International Journal of Applied Mathematics

Volume 30 No. $5 \quad 2017,375-386$

ISSN: $1311-1728$ (printed version); ISSN: 1314-8060 (on-line version)

doi: http://dx.doi.org/10.12732/ijam.v30i5.2

\title{
SINGLE MACHINE SLACK DUE-WINDOW SCHEDULING WITH LINEAR RESOURCE ALLOCATION, AGING EFFECT, AND A DETERIORATING RATE-MODIFYING ACTIVITY
}

\author{
Bo Cheng ${ }^{1}$, Ling Cheng ${ }^{2}$ \\ ${ }^{1}$ Department of Applied Mathematics \\ School of Finance \\ Guangdong University of Foreign Studies \\ Guangzhou, 510420, P.R. CHINA \\ ${ }^{2}$ School of Electrical and Information Engineering \\ University of the Witwatersrand \\ Private Bag 3, Wits. 2050 \\ Johannesburg, SOUTH AFRICA
}

\begin{abstract}
In this paper, we consider the slack due-window method and investigate single machine scheduling with a deteriorating rate-modifying activity, linear resource allocation and aging effect. The objective is to minimize the total cost caused by the due-window location, the due-window size, the earliness and tardiness with respect to a slack due-window, and resource consumption. We provide a polynomial-time algorithm to solve the corresponding problem.
\end{abstract}

AMS Subject Classification: 90B35

Key Words: aging effect, due-window, rate-modifying activity, resource allocation, scheduling

\section{Introduction}

In the studies on operations management, a good customer service usually requires jobs completed as close as possible to their due-dates. A time interval is assigned in the supply contract so that a job finished within this period will

Received: August 8, 2017

(C) 2017 Academic Publications

${ }^{\S}$ Correspondence author 
be considered on time and not be penalized. This period is called the duewindow of a job (cf. [1, 2]). The due-window assignment methods include common due-window, slack due-window (also called common flow allowance) and others.

Scheduling theory for jobs with changeable job processing times has been developed steadily in the last decade. The single machine common due-window assignment problem with deteriorating jobs and learning effect was studied by [3] and polynomial-time algorithms were given to minimize the total costs for earliness, tardiness, window location and window size. The parallel problem for the slack due-window model was investigated by [4]. Other scheduling problems with the aging effect were studied by $[5,6,7]$.

The effect of allocating additional resources on job processing times was also extensively investigated by $[8,9,10]$ and others. A linear function taking the amount of allocated resource as the input parameter was proposed to quantify the effect of additional resources on job processing times. For example, in a linear resource consumption model, the actual processing time of a job is determined by

$$
\tilde{p}=p-c u, \quad 0 \leq u \leq \bar{u}<\frac{p}{c},
$$

where $\tilde{p}$ is the actual processing time, $p$ is the non-compressed (normal) processing time, $c$ is the positive compression factor, $u$ is the actual resource allocated to the job, and $\bar{u}$ is the maximum resource that could be allocated to the job. For the linear resource consumption model in this paper, we remove the upper bound on $\bar{u}$ in (1), since the new one without the upper bound is more general.

Machine scheduling with a rate-modifying activity (RMA) was initially investigated by [11]. In this paper, at most one RMA can be scheduled before or after any job except that it is unnecessary to schedule a RMA after the last job. The scheduler decides when to perform the RMA. However, the RMA cannot interrupt any job. It means the RMA can be scheduled either before or after a job.

The combinations of the above-mentioned settings have been further investigated. [12] studied common due-window assignment and scheduling with time-dependent deteriorating jobs and a maintenance activity, and $[9,10]$ investigated the scheduling problem with resource allocation, aging effect and a deteriorating RMA based on common due-window method. [13] considered the slack due-window assignment and scheduling taking into account variable processing-time jobs and a rate-modifying activity.

In this paper, we discuss the problem of slack due-window assignment and single machine scheduling taking into account linear resource allocation and 
position-dependent deteriorating jobs with a RMA. To our best knowledge, this problem has not been studied in literatures.

The rest of this paper is organized as follows. A description of the problem under study is given in Section 2. In Section 3 a few important lemmas and properties are presented. In the same section, a polynomial-time solution is given. A numerical example is presented to demonstrate the polynomial-time solution in Section 4. The research is concluded and future study is foreseen in the last section.

\section{Model Formulation}

In the models considered in this paper, $n$ independent and non-preemptive jobs $J_{1}, J_{2}, \ldots, J_{n}$ and at most one RMA are scheduled on a single machine. All the jobs and the RMA can be scheduled at time zero. Let $p_{j}$ be the normal processing time of job $J_{j}$. The predetermined parameters of job $J_{j}$ are the job-dependent aging factor $a_{j}$ and the maximum available resource amount $\bar{u}_{j}$. Let $p_{j r}$ denote the actual processing time of $J_{j}$ scheduled in the $r$ th position. The actual resource allocated to job $J_{j}$ is denoted as $u_{j}$. For the linear resource consumption model, the actual processing time of job $J_{j}$ is determined by

$$
p_{j r}=p_{j} r^{a_{j}}-c_{j} u_{j},
$$

where $c_{j}$ is the positive compression rate of job $J_{j}, 0 \leq u_{j} \leq \bar{u}_{j}$ and $p_{j r} \geq 0$.

The RMA duration is determined by $f(t)=b+\sigma t$, where $b>0$ is the basic RMA time, $\sigma \geq 0$ is the RMA deterioration rate, and $t$ is the starting time of the RMA. The modifying rate of job $J_{j}$ is notated by $\lambda_{j}$. After the RMA, the machine will revert to its initial conditions, machine deterioration will start anew, and the processing time of job $J_{j}$ will be multiplied by $\lambda_{j}$. In the proposed model, we assume the RMA can improve the efficiency of a job (therefore $\lambda_{j}$ takes a value in $\left.(0,1]\right)$.

The due-window of job $J_{j}$ is specified by a pair of non-negative real numbers $\left[d_{j}, d_{j}^{\prime}\right]$ such that $d_{j} \leq d_{j}^{\prime}$, where $d_{j}$ and $d_{j}^{\prime}$ are the beginning and ending times of the due-window respectively. For the slack due-window method, $d_{j}$ and $d_{j}^{\prime}$ are determined by

$$
d_{j}=p_{j r}+q,
$$

and

$$
d_{j}^{\prime}=p_{j r}+q^{\prime}
$$

where $q^{\prime}>q$ are two job-independent constants. 
Then the due-window size $D_{j}=d_{j}-d_{j}^{\prime}=q^{\prime}-q$, for $j=1, \ldots, n$, is identical for all the jobs. Let $D=D_{j}$.

For a given schedule $\pi, C_{j}$ denotes the completion time of job $J_{j}, E_{j}=$ $\max \left\{0, d_{j}-C_{j}\right\}$ is the earliness value of job $J_{j}$, and $T_{j}=\max \left\{0, C_{j}-d_{j}^{\prime}\right\}$ is the tardiness value of job $J_{j}$. To this end, we can create the following total cost function

$$
Z=\sum_{j=1}^{n}\left(\alpha E_{j}+\beta T_{j}+\gamma d_{j}+\delta D\right)+\theta \sum_{j=1}^{n} G_{j} u_{j},
$$

which takes into account (i) earliness $E_{j}$, (ii) tardiness $T_{j}$, (iii) the starting time of the due-window $d_{j}$, (iv) the due-window size $D$, and (v) the resource allocation. We further define $\alpha>0, \beta>0, \gamma>0$ and $\delta>0$ representing the earliness, tardiness, due-window starting time and due-window size costs per unit time respectively. For the resource consumption cost, $G_{j}$ is defined as the per unit resource cost for job $J_{j}$ and $\theta$ is a constant weight which is specified by the decision-maker.

The general objective is to determine the optimal job sequence, the optimal location of the RMA, the optimal resource consumption and the optimal $q$ and $q^{\prime}$ to minimize the total cost function

$$
Z=Z\left(\pi, u, q, q^{\prime}\right)=\sum_{j=1}^{n}\left(\alpha E_{j}+\beta T_{j}+\gamma d_{j}+\delta D\right)+\theta \sum_{j=1}^{n} G_{j} u_{j} .
$$

The problems under study are denoted as

$$
\begin{gathered}
1 \mid S L K, p_{j r}=p_{j} r^{a_{j}}-c_{j} u_{j}, \\
R M A \mid \sum_{j=1}^{n}\left(\alpha E_{j}+\beta T_{j}+\gamma d_{j}+\delta D\right)+\theta \sum_{j=1}^{n} G_{j} u_{j},
\end{gathered}
$$

where $S L K$ and $R M A$ denote the slack due-window method and rate-modifying activity, respectively.

\section{Optimal Solution}

In this section some properties for an optimal schedule are obtained.

The proofs of the following lemmas are similar to those in [14] and [15]. We use a conventional notation $[r]$ to indicate the index of a job which is allocated at the $r$ th position. 
Lemma 1. If $C_{[r]} \geq d_{[r]}^{\prime}$ holds, then $C_{[r+1]} \geq d_{[r+1]}^{\prime}$.

Lemma 2. If $C_{[r]} \leq d_{[r]}$ holds, then $C_{[r-1]} \leq d_{[r-1]}$.

Consider a job sequence $\pi$ and a resource consumption way $u=\left(u_{1}, u_{2}, \ldots\right.$, $\left.u_{n}\right)$. Assume that $C_{[s]} \leq q \leq C_{[s+1]}$ and $C_{[t]} \leq q^{\prime} \leq C_{[t+1]}$. Then the total cost $Z$ is a linear function of $q$ and $q^{\prime}$, and thus an optimum is obtained either at $q=C_{[s]}$ or $q=C_{[s+1]}$ and either at $q^{\prime}=C_{[t]}$ or $q^{\prime}=C_{[t+1]}$.

Lemma 3. (i) For any given job sequence $\pi$ and resource consumption $u$, there exists an optimal schedule in which the values of $q$ and $q^{\prime}$ coincide with the completion times of the $k$-th and $l$-th jobs $(l \geq k)$ in the sequence.

(ii) An optimal schedule starts at time zero and contains no idle time between consecutive jobs.

For a number $a$, thesymbol $\lfloor a\rfloor$ denotes the largest integer not more than $a$.

Lemma 4. $k=\left\lfloor\frac{n(\delta-\gamma)}{\alpha}\right\rfloor$ and $l=\left\lfloor\frac{n(\beta-\delta)}{\beta}\right\rfloor$.

By Lemma 4 , the values of $k$ and $l$ can be calculated. Let $i$ be the position of the last job preceding the RMA. If the position of the RMA is before $k$ (i.e., $i<k$ ), then the total cost is given by

$$
\begin{aligned}
Z & =\sum_{r=1}^{n}\left(\alpha E_{[r]}+\beta T_{[r]}+\gamma d_{[r]}+\delta D\right)+\theta \sum_{j=1}^{n} G_{j} u_{j} \\
& =\alpha \sum_{r=1}^{k}\left(p_{j r}+q-C_{[r]}\right)+\beta \sum_{r=l+1}^{n}\left(C_{[r]}-p_{j r}-q^{\prime}\right) \\
& +\gamma \sum_{r=1}^{n}\left(q+p_{j r}\right)+n \delta\left(q^{\prime}-q\right)+\theta \sum_{j=1}^{n} G_{j} u_{j} \\
& =\alpha \sum_{r=1}^{k} j p_{j r}+\alpha i\left(b+\sigma \sum_{r=1}^{i} p_{j r}\right)+\beta \sum_{r=l+1}^{n}(n-r) p_{j r} \\
& +\gamma\left(n\left(b+\sigma \sum_{r=1}^{i} p_{j r}\right)+(n+1) \sum_{r=1}^{k} p_{j r}+\sum_{r=k+1}^{n} p_{j r}\right)
\end{aligned}
$$




$$
\begin{aligned}
& +n \delta \sum_{r=k+1}^{l} p_{j r}+\theta \sum_{j=1}^{n} G_{j} u_{j} \\
& =n b \gamma+\alpha i b+\sum_{r=1}^{n} w_{r} p_{j r}+\theta \sum_{j=1}^{n} G_{j} u_{j},
\end{aligned}
$$

where

$$
w_{r}= \begin{cases}\alpha r+\alpha i \sigma+\gamma n \sigma+(n+1) \gamma, & \text { if } r=1,2, \ldots, i \\ \alpha r+(n+1) \gamma, & \text { if } r=i+1, i+2, \ldots, k, \\ \gamma+n \delta, & \text { if } r=k+1, k+2, \ldots, l, \\ \beta(n-r)+\gamma, & \text { if } r=l+1, l+2, \ldots, n .\end{cases}
$$

If $k \leq i<l$, then we have

$$
\begin{aligned}
Z & =\alpha \sum_{r=1}^{k} r p_{j r}+\beta \sum_{r=l+1}^{n}(n-r) p_{j r}+\gamma\left((n+1) \sum_{r=1}^{k} p_{j r}+\sum_{r=k+1}^{n} p_{j r}\right) \\
& +n \delta\left(b+\sigma \sum_{r=1}^{i} p_{j r}\right)+n \delta \sum_{r=k+1}^{l} p_{j r}+\theta \sum_{j=1}^{n} G_{j} u_{j} \\
& =n \delta b+\sum_{r=1}^{n} w_{r} p_{j r}+\theta \sum_{j=1}^{n} G_{j} u_{j},
\end{aligned}
$$

where

$$
w_{r}= \begin{cases}\alpha r+\gamma(n+1)+n \delta \sigma, & 1 \leq r \leq k \\ \gamma+n \delta \sigma+n \delta, & k<r \leq i \\ \gamma+n \delta, & i<r \leq l \\ \beta(n-r)+\gamma, & l<r \leq n\end{cases}
$$

If $l \leq i \leq n$, then we have

$$
\begin{aligned}
Z & =\alpha \sum_{r=1}^{k} r p_{j r}+\beta\left(\sum_{r=l+1}^{n}(n-r) p_{j r}+(n-i)\left(b+\sigma \sum_{r=1}^{i} p_{j r}\right)\right) \\
& +\gamma\left((n+1) \sum_{r=1}^{k} p_{j r}+\sum_{r=k+1}^{n} p_{j r}\right)+n \delta \sum_{r=k+1}^{l} p_{j r}+\theta \sum_{j=1}^{n} G_{j} u_{j} \\
& =(n-i) \beta b+\sum_{r=1}^{n} w_{r} p_{j r}+\theta \sum_{j=1}^{n} G_{j} u_{j},
\end{aligned}
$$


where

$$
w_{r}= \begin{cases}\alpha r+\beta(n-i) \sigma+\gamma(n+1), & 1 \leq r \leq k, \\ \beta(n-i) \sigma+\gamma+n \delta, & k<r \leq l, \\ \beta(n-r)+\beta(n-i) \sigma+\gamma, & l<r \leq i, \\ \beta(n-r)+\gamma, & i<r \leq n .\end{cases}
$$

Note that when $i=n$ there is no RMA scheduled since by then all jobs are finished.

For the linear resource consumption models, we have

$$
p_{j r}= \begin{cases}p_{j} r^{a_{j}}-c_{j} u_{j}, & r \leq i, \\ \lambda_{j} p_{j}(r-i)^{a_{j}}-c_{j} u_{j}, & r>i,\end{cases}
$$

and thus

$$
Z=M+\sum_{r=1}^{n} w_{r} p_{j} \eta_{j r}+\sum_{r=1}^{n}\left(\theta G_{j}-w_{r} c_{j}\right) u_{j}
$$

where

$$
M= \begin{cases}n b \gamma+\alpha i b, & i<k \\ n \delta b, & k \leq i<l \\ (n-i) \beta b, & l \leq i \leq n\end{cases}
$$

and

$$
\eta_{j r}= \begin{cases}r^{a_{j}}, & r \leq i, \\ \lambda_{j}(r-i)^{a_{j}}, & r>i .\end{cases}
$$

Therefore, minimizing the objective function $Z$ is equivalent to minimizing $Z^{\prime}$ :

$$
Z^{\prime}=\sum_{r=1}^{n} w_{r} p_{j} \eta_{j r}+\sum_{r=1}^{n}\left(\theta G_{j}-w_{r} c_{j}\right) u_{j} .
$$

Since $p_{j r}>0$, we have

$$
p_{j} r^{a_{j}}-c_{j} u_{j} \geq 0 \text { if } r \leq i
$$

and

$$
\lambda_{j} p_{j}(r-i)^{a_{j}}-c_{j} u_{j} \geq 0 \text { if } r>i .
$$

Set

$$
u_{j}^{\prime}= \begin{cases}\min \left\{\bar{u}_{j}, \frac{p_{j} r^{a_{j}}}{c_{j}}\right\}, & \text { if } r \leq i, \\ \min \left\{\bar{u}_{j}, \frac{\lambda_{j} p_{j}(r-i)^{a_{j}}}{c_{j}}\right\}, & \text { if } r>i .\end{cases}
$$


Therefore, we have $u_{j} \leq u_{j}^{\prime}$.

We see the optimal resource consumption for a job depends on the sign of $\theta G_{j}-w_{r} c_{j}$. Let $u_{j}^{*}$ be the optimal resource consumption for job $J_{j}$. Then,

$$
u_{j}^{*}= \begin{cases}u_{j}^{\prime}, & \text { if } \theta G_{j}-w_{r} c_{j}<0 \\ u_{j} \in\left[0, u_{j}^{\prime}\right], & \text { if } \theta G_{j}-w_{r} c_{j}=0 \\ 0, & \text { if } \theta G_{j}-w_{r} c_{j}>0\end{cases}
$$

To this end we can define the element $\chi_{j r}$ in an assignment matrix as follows:

$$
\chi_{j r}= \begin{cases}w_{r} p_{j} \eta_{j r}, & \text { if } \theta G_{j}-w_{r} c_{j} \geq 0 \\ w_{r} p_{j} \eta_{j r}+\left(\theta G_{j}-w_{r} c_{j}\right) u_{j}^{\prime}, & \text { if } \theta G_{j}-w_{r} c_{j}<0\end{cases}
$$

and

$$
z_{j r}= \begin{cases}1 & \text { if job } J_{j} \text { is scheduled in the } r \text { th position } \\ 0 & \text { otherwise. }\end{cases}
$$

To minimize the problem $1\left|S L K, p_{j r}=p_{j} r^{a_{j}}-c_{j} u_{j}, R M A\right| \sum_{j=1}^{n}\left(\alpha E_{j}+\beta T_{j}+\right.$ $\left.\gamma d_{j}+\delta D\right)+\theta \sum_{j=1}^{n} G_{j} u_{j}$ is equivalent to minimizing the following Assignment Problem, which can be solved in time complexity $O\left(n^{3}\right)$ :

$$
\begin{aligned}
& \min \sum_{j=1}^{n} \sum_{r=1}^{n} \chi_{j r} z_{j r} \\
& \text { s.t. } \begin{cases}\sum_{j=1}^{n} z_{j r}=1, & r=1,2, \ldots, n, \\
\sum_{r=1}^{n} z_{j r}=1, & j=1,2, \ldots, n, \\
z_{j r}=0 \text { or } 1, & j, r=1,2, \ldots, n .\end{cases}
\end{aligned}
$$

Here (24) presents a 0-1 integer linear programming problem, which guarantees that each position has one job scheduled and each job is scheduled once. The following solution algorithm has an $O\left(n^{4}\right)$ time complexity.

Algorithm 1. Solution algorithm for the problem $1 \mid S L K, p_{j r}=p_{j} r^{a_{j}}-$ $c_{j} u_{j}, R M A \mid \sum_{j=1}^{n}\left(\alpha E_{j}+\beta T_{j}+\gamma d_{j}+\delta D\right)+\theta \sum_{j=1}^{n} G_{j} u_{j}$.

$1 \quad$ SET $k=\left\lfloor\frac{n(\delta-\gamma)}{\alpha}\right\rfloor, l=\left\lfloor\frac{n(\beta-\delta)}{\beta}\right\rfloor$.

2 FOR each position $i=0,1, \ldots, n$ available to allocate rate-modifying activity

3 FOR each position $r=1,2, \ldots, n$ in a schedule 
DETERMINE the positional weight $w_{r}$ END FOR

12 END FOR

FOR each position $r=1,2, \ldots, n$ in a schedule END FOR

DETERMINE the value $\chi_{j r}$ according to $(22)$

END FOR

DETERMINE a local optimal schedule of the assignment problem described in (24) and its total cost

13 DETERMINE the global optimal schedule with the minimum total cost

Theorem 1. Algorithm 1 solves the problem $1 \mid S L K, p_{j r}=p_{j} r^{a_{j}}-c_{j} u_{j}$, $R M A \mid \sum_{j=1}^{n}\left(\alpha E_{j}+\beta T_{j}+\gamma d_{j}+\delta D\right)+\theta \sum_{j=1}^{n} G_{j} u_{j}$ in $O\left(n^{4}\right)$ time.

Proof. The correction of Algorithm 1 is guaranteed by Lemmas 4 and the derivation from $(7)$ to $(24)$. The time complexity from Step 3 to Step 11 is $O\left(n^{3}\right)$. In the outer loop from Step 2 to Step 12, position index $i$ takes on integer values between 0 to $n$. Hence, the time complexity for solving the $1\left|S L K, p_{j r}=p_{j} r^{a_{j}}-c_{j} u_{j}, R M A\right| \sum_{j=1}^{n}\left(\alpha E_{j}+\beta T_{j}+\gamma d_{j}+\delta D\right)+\theta \sum_{j=1}^{n} G_{j} u_{j}$ problem is $O\left(n^{4}\right)$.

In most studies of the linear resource model, an upper bound on $\bar{u}$ is stated as (1). In this paper we remove this constraint and consider a more general case, to which Algorithm 1 gives a solution.

\section{Numerical Example}

In this section, Algorithm 1 for the linear resource model is demonstrated by the following example.

Example 1. There are $n=7$ jobs. The initial setup of all jobs is illustrated by Table 1 .

The penalties for unit earliness, tardiness, due-window starting time and due-window size are $\alpha=2, \beta=18, \gamma=4$ and $\delta=5$, respectively. The basic maintenance time is $b=15$ and the deteriorating maintenance factor is $\sigma=0.1$. The constant weight for resource consumption $\theta=0.8$. 


\begin{tabular}{cccccccc}
\hline$j$ & 1 & 2 & 3 & 4 & 5 & 6 & 7 \\
\hline$p_{j}$ & 45 & 18 & 48 & 46 & 50 & 43 & 21 \\
$a_{j}$ & 0.3 & 0.5 & 0.25 & 0.3 & 0.15 & 0.4 & 0.2 \\
$c_{j}$ & 3 & 2 & 3 & 4 & 3 & 4 & 2 \\
$\lambda_{j}$ & 0.58 & 0.84 & 0.45 & 0.55 & 0.38 & 0.64 & 0.75 \\
$G_{j}$ & 34 & 40 & 25 & 38 & 24 & 48 & 42 \\
$\bar{u}_{j}$ & 99 & 7 & 7 & 6 & 6 & 6 & 7 \\
\hline
\end{tabular}

Table 1: The settings in Example 1.

Solution: By Lemma 4, we have the locations of $k=\left\lfloor\frac{n(\delta-\gamma)}{\alpha}\right\rfloor=3$ and $l=\left\lfloor\frac{n(\beta-\delta)}{\beta}\right\rfloor=5$.

As shown in Table 2, all the local optimal job sequences and the corresponding total costs are presented, among which the optimal total cost is underlined. The global optimal solution for this example includes the following: (i) the job sequence is $(2,4,3,5,1,7,6)$ and the corresponding job starting time and actual processing time are $(0,19.40,20.70,25.39,29.79,29.79,37.52)$ and $(4.00$, $1.30,4.69,4.40,0,7.73,56.35)$, respectively; (ii) the slack window parameters are $q=25.39$ and $q^{\prime}=29.79$; (iii) the RMA is located immediately after the first job (i.e. Job $J_{2}$ ), starting at time $t=4.00$ and ending at time $t=19.40$ (maintenance duration 15.40); (iv) the optimal resource consumption of each job is $(13.19,7.00,7.00,6.00,6.00,0,7.00)$; (v) the total cost is $Z=2645.61$.

\begin{tabular}{ccc}
\hline$i$ & Job sequence & $Z$ \\
\hline 0 & $(2,4,3,5,1,7,6)$ & 2915.80 \\
1 & $(2,4,3,5,1,7,6)$ & $\underline{2645.61}$ \\
2 & $(2,1,4,3,5,7,6)$ & 2790.61 \\
3 & $(2,7,1,4,3,5,6)$ & 3060.96 \\
4 & $(6,2,7,1,3,5,4)$ & 3989.78 \\
5 & $(6,4,2,7,1,3,5)$ & 5616.00 \\
6 & $(6,4,2,7,1,5,3)$ & 6290.07 \\
7 & $(6,4,2,7,1,5,3)$ & 6012.09 \\
\hline
\end{tabular}

Table 2: The corresponding local optimal job sequences and total costs with one RMA at all possible positions in Example 1. 


\section{Conclusion}

We have investigated a single machine scheduling and slack due-window assignment problem with linear resource allocation, aging effect and a deteriorating rate-modifying maintenance activity. The objective is to minimize the total cost caused by the due-window location, due-window size, earliness, tardiness and resource consumption.

Further research may consider the problem with other performance measures, or the problem with multiple rate-modifying maintenance activities.

\section{References}

[1] T. C. E. Cheng, Optimal common due-date with limited completion time deviation, Computers \& Operations Research, 15 (1988), 91-96.

[2] S. Liman, S. Panwalkar, and S. Thongmee, Common due window size and location determination in a single machine scheduling problem, Journal of the Operational Research Society, 49 (1998), 1007-1010.

[3] J.-B. Wang and C. Wang, Single-machine due-window assignment problem with learning effect and deteriorating jobs, Applied Mathematical Modelling, 35 (2011), 4017-4022.

[4] J.-B. Wang, L. Liu, and C. Wang, Single machine SLK/DIF due window assignment problem with learning effect and deteriorating jobs, Applied Mathematical Modelling, 37 (2013), 8394-8400.

[5] S.-J. Yang, D.-L. Yang, and T. C. E. Cheng, Single-machine due-window assignment and scheduling with job-dependent aging effects and deteriorating maintenance, Computers \& Operations Research, 37 (2010), 15101514.

[6] C.-L. Zhao and H.-Y. Tang, Single machine scheduling with general jobdependent aging effect and maintenance activities to minimize makespan, Applied Mathematical Modelling, 34 (2010), 837-841.

[7] R. Rudek, Scheduling problems with position dependent job processing times: computational complexity results, Annals of Operations Research, 196 (2012), 491-516.

[8] R. Vickson, Two single machine sequencing problems involving controllable job processing times, AIIE Transactions, 12 (1980), 258-262. 
[9] M. Ji, J. Ge, K. Chen, and T. Cheng, Single-machine due-window assignment and scheduling with resource allocation, aging effect, and a deteriorating rate-modifying activity, Computers \& Industrial Engineering, 66 (2013), 952-961.

[10] B. Cheng and L. Cheng, Note on single-machine due-window assignment and scheduling with resource allocation, aging effect, and a deteriorating rate-modifying activity, Computers \& Industrial Engineering, 78 (2014), $320-322$.

[11] C.-Y. Lee and V. Leon, Machine scheduling with a rate-modifying activity, European Journal of Operational Research, 128 (2001), 119-128.

[12] T. C. E. Cheng, S.-J. Yang, and D.-L. Yang, Common due-window assignment and scheduling of linear time-dependent deteriorating jobs and a deteriorating maintenance activity, International Journal of Production Economics, 135 (2012), 154-161.

[13] B. Mor and G. Mosheiov, Scheduling a maintenance activity and duewindow assignment based on common flow allowance, International Journal of Production Economics, 135 (2012), 222-230.

[14] G. Mosheiov and D. Oron, Job-dependent due-window assignment based on a common flow allowance, Foundations of Computing and Decision Sciences, 35 (2010), 185-195.

[15] K. Chen, M. Ji, and J. Ge, A note on scheduling a maintenance activity and due-window assignment based on common flow allowance, International Journal of Production Economics, 145 (2013), 645-646. 